\title{
Revision of the Structures Assigned to the Fungal Metabolites Boletunone A and B
}

Wolfgang Steglich* and Veronika Hellwig

\section{Supporting Information}

\section{NMR data}

Table 2. NMR data reported for boletunone B in ref 1 assigned to revised structure 6

\begin{tabular}{cll}
\hline no. $^{a}$ & $\delta_{\mathrm{C}}$ & \multicolumn{1}{c}{\begin{tabular}{c}
\multicolumn{1}{c}{$\delta_{\mathrm{H}}$} \\
$($ mult $, J, \mathrm{~Hz})$
\end{tabular}} \\
\hline 1 & 173.0 & $4.34(\mathrm{~d}, 3.6)$ \\
2 & 77.4 & $2.57(\mathrm{~m})$ \\
3 & 46.0 & $2.08(\mathrm{~m})$ \\
4 & 27.6 & $\mathrm{H}_{\alpha} 3.35(\mathrm{dd}, 11.5,6.8)$ \\
5 & 66.6 & $\mathrm{H}_{\beta} 4.10(\mathrm{dd}, 11.5,11.5)$ \\
& & $3.77(\mathrm{~s})$ \\
6 & 54.5 & \\
7 & 71.9 & \\
8 & 198.6 & $6.77(\mathrm{br} . \mathrm{s})$ \\
9 & 131.7 & $\mathrm{H}_{\alpha} 2.56(\mathrm{~d}, 19.7)$ \\
10 & 145.5 & $\mathrm{H}_{\beta} 2.50(\mathrm{~d}, 19.7)$ \\
11 & 30.1 & \\
& & $0.94(\mathrm{~s}, 3 \mathrm{H})$ \\
12 & 107.0 & $0.83(\mathrm{~d}, 7.1,3 \mathrm{H})$ \\
13 & 21.0 & $1.63(\mathrm{~s}, 3 \mathrm{H})$ \\
14 & 15.0 & \\
15 & 15.2 & \\
& & \\
${ }^{a}$ Cyclocalopin numbering (see Figure 1). Assignments \\
of $\mathrm{H}_{\alpha}$ and $\mathrm{H}_{\beta}$ are opposite to those given in ref 1.
\end{tabular}

Comparison of NOE and HMBC correlations between the old and revised structures 
Table 3. NOE correlations given for boletunone A in ref 1 assigned to revised structure 5

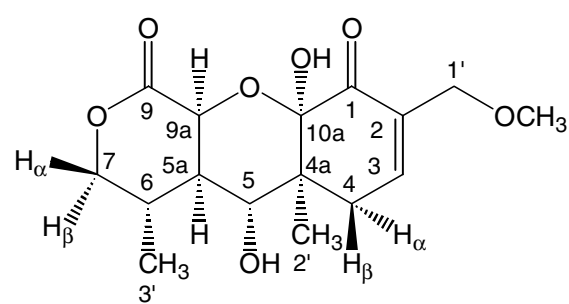

Structure 1, proposed in Org. Lett.. 2004, 6, 823-826

(Original numbering)

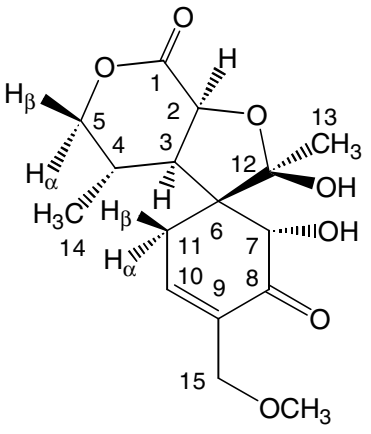

Revised structure 5 :

15-methoxycyclocalopin A

(Cyclocalopin numbering)

\begin{tabular}{|c|c|c|c|c|}
\hline \multicolumn{2}{|c|}{$\begin{array}{l}\text { NOE correlations given } \\
\text { in ref } 1 \text { for structure } \mathbf{1}^{a} \\
\end{array}$} & \multicolumn{2}{|c|}{$\begin{array}{l}\text { Corresponding NOEs in revised } \\
\text { structure } \mathbf{5}^{b}\end{array}$} & $\begin{array}{l}\text { Were these NOEs } \\
\text { observed for } \mathbf{3} \text { in ref } 2 \text { ? }\end{array}$ \\
\hline $4-\mathrm{H}_{\alpha}$ & $\leftrightarrow \quad 2^{\prime}-\mathrm{Me}$ & $11-\mathrm{H}_{\beta}$ & $\leftrightarrow 13-\mathrm{Me}$ & yes \\
\hline $2^{\prime}-\mathrm{Me}$ & $\leftrightarrow \quad 10 \mathrm{a}-\mathrm{OH}$ & 13-Me & $\leftrightarrow \quad 12-\mathrm{OH}$ & \\
\hline $4-\mathrm{H}_{\beta}$ & $\leftrightarrow \quad 5-\mathrm{H}(?)$ & $11-\mathrm{H}_{\alpha}$ & $\leftrightarrow \quad 7-\mathrm{H}(\text { not possible! })^{c}$ & \\
\hline $4-\mathrm{H}_{\beta}$ & $\leftrightarrow \quad 6-\mathrm{H}$ & $11-\mathrm{H}_{\alpha}$ & $\leftrightarrow 4-\mathrm{H}$ & yes \\
\hline $9 \mathrm{a}-\mathrm{H}$ & $\leftrightarrow 5 \mathrm{a}-\mathrm{H}$ & $2-\mathrm{H}$ & $\leftrightarrow 3-\mathrm{H}$ & yes \\
\hline $5 \mathrm{a}-\mathrm{H}$ & $\leftrightarrow 7-\mathrm{H}_{\beta}$ & $3-\mathrm{H}$ & $\leftrightarrow 5-\mathrm{H}_{\alpha}$ & yes \\
\hline $6-\mathrm{H}$ & $\leftrightarrow \quad 7-\mathrm{H}_{\alpha}$ & $4-\mathrm{H}$ & $\leftrightarrow 5-\mathrm{H}_{\beta}$ & \\
\hline 3'-Me & $\leftrightarrow \quad 7-\mathrm{H}_{\alpha}$ & 14-Me & $\leftrightarrow 5-\mathrm{H}_{\beta}$ & \\
\hline $3^{\prime}-\mathrm{Me}$ & $\leftrightarrow 7-\mathrm{H}_{\beta}$ & 14-Me & $\leftrightarrow 5-\mathrm{H}_{\alpha}$ & \\
\hline
\end{tabular}

Table 4. HMBC correlations given for boletunone A in ref 1 assigned to revised structure 5.

\begin{tabular}{|c|c|}
\hline $\begin{array}{l}\text { HMBC correlations given in } \\
\text { ref } 1 \text { for structure } \mathbf{1}^{a}\end{array}$ & $\begin{array}{l}\text { Corresponding } \mathrm{HMBCs} \text { in } \\
\text { revised structure } \mathbf{5}^{b}\end{array}$ \\
\hline$\rightarrow \mathrm{C}-1, \mathrm{C}-4, \mathrm{C}-4 \mathrm{a}$ & $\rightarrow$ C-8, C-11, C-6 \\
\hline $4-\mathrm{H}_{\beta} \rightarrow \mathrm{C}-4 \mathrm{a}, \mathrm{C}-10 \mathrm{a}, \mathrm{C}-5$ & $\rightarrow \mathrm{C}-6, \mathrm{C}-12, \mathrm{C}-7$ \\
\hline $5-\mathrm{H}^{\beta} \rightarrow \mathrm{C}-1, \mathrm{C}-4 \mathrm{a}, \mathrm{C}-10 \mathrm{a}$ & $\rightarrow \mathrm{C}-8, \mathrm{C}-6, \mathrm{C}-12$ \\
\hline $5 \mathrm{a}-\mathrm{H} \rightarrow \mathrm{C}-9$ & $\rightarrow \mathrm{C}-1$ \\
\hline $7-\mathrm{H}_{\alpha} \rightarrow \mathrm{C}-9$ & $\rightarrow \mathrm{C}-1$ \\
\hline $9 \mathrm{a}-\mathrm{H} \rightarrow \mathrm{C}-9$ & $\rightarrow \mathrm{C}-1$ \\
\hline
\end{tabular}

${ }^{a}$ Numbering as in ref $1 .{ }^{b}$ Cyclocalopin numbering. Assignments 
of $\mathrm{H}_{\alpha}$ and $\mathrm{H}_{\beta}$ are opposite to those given in ref 2 .

Table 5. NOE correlations given for boletunone B in ref 1 assigned to revised structure 6

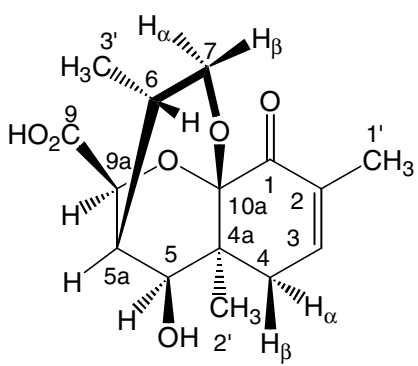

Structure 2, proposed in Org. Lett.. 2004, 6, 823-826

(Original numbering)

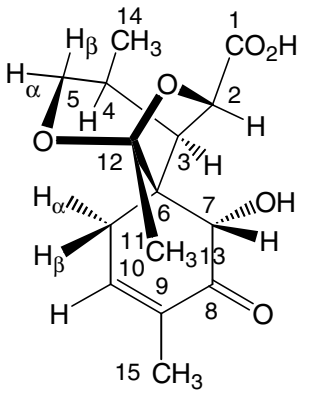

Revised structure 6 : isocyclocalopin A

(Cyclocalopin numbering)

\begin{tabular}{|c|c|c|c|c|c|}
\hline \multicolumn{2}{|c|}{$\begin{array}{l}\text { NOE correlations given } \\
\text { in ref } 1 \text { for structure } \mathbf{2}^{a}\end{array}$} & \multicolumn{3}{|c|}{$\begin{array}{l}\text { Corresponding NOEs in } \\
\text { revised structure } \mathbf{6}^{b} \\
\end{array}$} & \multirow[t]{2}{*}{$\begin{array}{l}\text { Were these NOEs observed } \\
\text { for } \mathbf{3} \text { in ref } 2 \text { ? }\end{array}$} \\
\hline $4-\mathrm{H}_{\alpha}$ & $\leftrightarrow \quad 3-\mathrm{H}$ & $11-\mathrm{H}_{\beta}$ & $\leftrightarrow$ & $10-\mathrm{H}$ & \\
\hline $4-\mathrm{H}_{\alpha}$ & $\leftrightarrow \quad 2^{\prime}-\mathrm{Me}$ & $11-\mathrm{H}_{\beta}$ & $\leftrightarrow$ & 13-Me & yes \\
\hline $2^{\prime}-\mathrm{Me}$ & $\leftrightarrow \quad 5-\mathrm{H}$ & $13-\mathrm{Me}$ & $\leftrightarrow$ & $7-\mathrm{H}$ & yes \\
\hline $5-\mathrm{H}$ & $\leftrightarrow \quad 9 \mathrm{a}-\mathrm{H}$ & 7-H & $\leftrightarrow$ & 2-H & no \\
\hline 6-H & $\leftrightarrow \quad 7-\mathrm{H}_{\beta}$ & 4-H & $\leftrightarrow$ & $5-\mathrm{H}_{\alpha}$ & \\
\hline 6-H & $\leftrightarrow \quad 4-\mathrm{H}_{3}$ & $4-\mathrm{H}$ & $\leftrightarrow$ & $11-\mathrm{H}_{\alpha}$ & yes \\
\hline $3^{\prime}-\mathrm{Me}$ & $\leftrightarrow \quad 7-\mathrm{H}_{\alpha}$ & 14-Me & $\leftrightarrow$ & $5-\mathrm{H}_{\beta}$ & \\
\hline $3^{\prime}-\mathrm{Me}$ & $\leftrightarrow \quad 7-\mathrm{H}_{\beta}$ & 14-Me & $\leftrightarrow$ & $5-\mathrm{H}_{\alpha}$ & \\
\hline
\end{tabular}

Table 6. HMBC correlations given for boletunone B in ref 1 assigned to revised structure 6

\begin{tabular}{|c|c|}
\hline $\begin{array}{l}\text { HMBC correlations given in ref } 1 \text { for } \\
\text { structure } \mathbf{2}^{a}\end{array}$ & $\begin{array}{l}\text { Corresponding HMBCs in revised } \\
\text { structure } 6^{b}\end{array}$ \\
\hline $7-\mathrm{H}_{\beta} \rightarrow$ C-5a, C-6, C-10a; not C-9! & $5-\mathrm{H}_{\alpha} \rightarrow \mathrm{C}-3, \mathrm{C}-4, \mathrm{C}-12 ;$ not $\mathrm{C}-1 !$ \\
\hline
\end{tabular}

